\title{
Transient Reduction in Control Loops in Case of Joint Plant-Controller Reconfiguration
}

\author{
Gyula Simon, Tamás Kovácsházy, Gábor Péceli \\ Department of Measurement and Information Systems \\ Budapest University of Technology and Economics \\ 1521 Budapest, Hungary \\ Phone: +361463 2057, Fax: +3614634112 \\ Email: \{simon,khazy,peceli\}@ mit.bme.hu
}

\begin{abstract}
In control systems undesirable transients may occur when either the controller or the plant is reconfigured during the operation. This paper gives new solution for the transient reduction problem in simple feedback control loops to maintain the smooth steady-state behavior even if both the plant and the controller are changed simultaneously. The transients are reduced in least-squares sense by using an anti-transient signal.
\end{abstract}

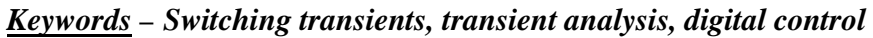

\section{INTRODUCTION}

Recent advances in sensor and information technology alleviate the development of control engineering applications for changing operating environments. The majority of the resulting solutions can be considered as hybrid dynamical systems [1], which combine continuous-state and discrete event dynamics. Such systems can be characterized by a number of distinct operational modes [2], [3]. If there is an "energy content" mismatch between the subsequent modes of the dynamical system, then reconfiguration transients are unavoidable [4]. The transients may be harmful, therefore undesirable, so they should be limited, or possibly reduced. Until now very few results are available in the control and signal processing literature concerning this issue. The problem is recognized, see, e.g. [6], but as a possible solution only the multiple-step reconfiguration is proposed. In some other works, see e.g. [7], transients are identified as convergence trajectories of adaptive schemes. In the signal processing literature some attempts were made to reduce the transients of variable filters [8]. Quite recently the case of controller reconfigurations was studied by the authors, and different solutions were proposed, e.g. choice of proper structures [5], and online transient management techniques [4].

This paper introduces novel transient control strategies for simple feedback loops, where, under certain conditions, the plant, or both the plant and the controller simultaneously, are switched to a new operational mode.

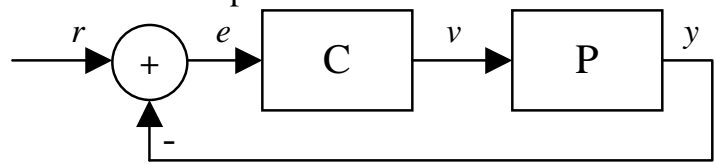

Figure 1. The feedback control loop system. C: controller, P: plant

\section{RECONFIGURATION TRANSIENTS IN CONTROL LOOPS}

In this paper transients in simple feedback control loops are investigated (see Fig.1). Further restriction is that only the steady state behavior is examined (i.e. the reference signal $r$ is constant and the system is in steady state when the reconfiguration happens).

The transients in control loops (see Fig.1) can happen due to three different sources:

(a) changing the reference; perturbations,

(b) controller (C) adaptation/reconfiguration,

(c) plant $(\mathrm{P})$ "reconfiguration" (mode changes, faults, degradation, recovery, repair).

Case (a) is handled by the properly chosen controller. Cases (b) and (c), however, can be considered as special disturbances that the designer may have information about, and the appropriate usage of this additional information may enable the effective reduction of the caused transients in certain cases. In this paper only transients due to reconfigurations (cases (b) and (c)) are considered.

If the controller (C) is reconfigured, and steady-state statevariable values of the controller are different before and after switching, then a transient phenomenon will appear, unless proper initialization of the state variables is performed [4]. State adjustment is an obvious alternative in the case of processor-based digital controllers where state variables are available and can easily be modified. A more serious situation is the plant $(\mathrm{P})$ reconfiguration, because usually the inner states of the plant are accessible and/or cannot be modified arbitrarily. For this very reason, in the majority of the cases, transients are unavoidable, and the rate of transient reduction relative to the open loop behavior depends completely on the properties of the closed loop. In a third scenario the plant $(\mathrm{P})$ and controller (C) are changed simultaneously. This is the most important, and of course, the most difficult situation. However, the information on the plant's reconfiguration gives the freedom to the designer to interfere with the transients caused by the plant reconfiguration, and possibly decrease 


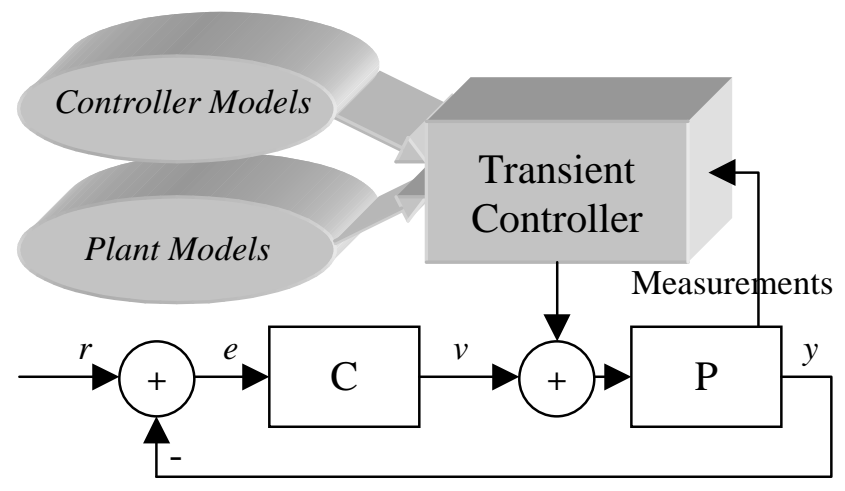

Fig. 2. Active transient control with anti-transient signal injection

them. In this paper the results concerning this case are introduced.

The "reconfiguration" of the plant can occur because

- the plant is changed deliberately (e.g., multi-modal operation), and thus the change is know a priori, or

- the plant is changed because of a sudden system fault or external event, and the change is detected only after its occurrence.

After plant "reconfiguration", to meet certain criteria, the reconfiguration of the controller might be required, as well. This controller reconfiguration should be carried out in a way that the reconfiguration of the controller does not induce additional transients, and with the reconfiguration of controller the plant's transients are also canceled or decreased. This first goal can be reached by the adequate initialization of the new controller [4], but this technique is not powerful enough to handle the plant's transients as well. For this purpose an extra anti-transient signal is proposed, which is produced by the Transient Controller (see Fig. 2.). This scheme can be considered as a feed-forward transient controller, which is modelbased; and can use the model of the controller and the plant. The operation of the Transient Controller in time can be $a$ priori or a posteriori.

- A posteriori transient control. Typical example is when an unexpected, but quickly detected fault occurs in the plant. The anti-transient injection starts after the plant's reconfiguration.

- A priori transient control. If the plant reconfiguration is known in advance (e.g. deliberate mode change) then the transient controller can start the operation before the plant itself is reconfigured.

\section{RECONFIGURATION STRATEGY}

The following constraints are supposed to be valid when the transient injection method is used:
- The system is in steady state when the reconfiguration happens

- The desired signal is constant and the control goal is to provide the smooth steady-state output even if the controller and the plant are changed.

- The controller's state variables can be modified, but the states of the plant cannot.

- The model of the plant (before and after the reconfiguration) is available. Note that in this paper a discrete statespace representation (approximation) of the plant is used.

- The plant and the controller are observable and controllable.

The proposed anti-transient signal design has the following main steps:

- Determine the state variables of the plant and the controller, and the values of $v$ and $y$ in steady state in the closed-loop system, for the given reference input $(r)$. Note that having the (observable and controllable) models of the plant and the controller, the steady-state values can easily be calculated [9].

- Calculate the injected control signal to minimize the effect of the plant's reconfiguration. The goal is to minimize the power of the transient as well as the state mismatch after the injection.

- Initialize the controller's states to provide smooth transition [4], neglecting the transients possibly caused by the plant's reconfiguration.

Notes:

- The proposed algorithm is not optimal in the sense that it handles the effects of the plant and controller reconfiguration separately.

- Although the transient suppression usually cannot be perfect, the closed control loop cancels the rest of the transients. The purpose of the anti-transient injection is to decrease the effect of the transients and speed up the cancellation.

\section{DESIGN OF THE ANTI-TRANSIENT SIGNAL}

The design of the anti-transient signal can be considered as an optimization problem, because the simultaneous minimization of the state variable mismatch and the plant output transients is to be achieved. The problem can be formulated as a leastsquares problem: The measure of the transient error is a sum of two components: the output error power during the time of the injection, and the state-mismatch power after the injection. The first component keeps the output error low during the injection, while the second component tries to minimize the state error at the end of the injection, and thus to provide low output error after the injection stopped. Since the a posteriori case can be considered as a special case of the a priori 
control, in the following the more general a priori antitransient injection will be described. The number of injected samples after the reconfiguration is $N_{2}$ (in both cases), while $N_{1}$ denotes the number of injected samples before the reconfiguration (in case of a priori anti-transient control).

The discrete state-space approximation of the system is

$$
\begin{gathered}
x_{k+1}=A x_{k}+B u_{k} \\
y_{k}=C x_{k}
\end{gathered}
$$

where $x, u, y$ are the state variables, the input, and the output, respectively. The error system after a reconfiguration can be described similarly:

$$
\begin{gathered}
\tilde{x}_{k+1}=A \tilde{x}_{k}+B u_{k} \\
\tilde{y}_{k}=C \tilde{x}_{k}
\end{gathered}
$$

where $\tilde{x}$ is the state error (i.e. the difference between the ideal and the actual states), and $\tilde{y}$ is the output error (i.e. the difference between the ideal steady-state output, and the actual output). The output error sequence can be expressed as a function of the input sequence:

$$
\mathbf{y}=\left[\begin{array}{cc}
\Xi_{1} & \mathbf{0} \\
\Phi & \Xi_{2}
\end{array}\right] \mathbf{u}+\left[\begin{array}{cc}
\Theta_{1} & \mathbf{0} \\
\Psi & \Theta_{2}
\end{array}\right]\left[\begin{array}{c}
\tilde{x}_{01} \\
\Delta x_{i d}
\end{array}\right]
$$

where

$$
\mathbf{y}=\left[\begin{array}{c}
\tilde{y}_{1} \\
\tilde{y}_{2} \\
\mathrm{M} \\
\tilde{y}_{N_{1}} \\
\tilde{y}_{N_{1}+1} \\
\tilde{y}_{N_{1}+2} \\
\mathrm{M} \\
\tilde{y}_{N_{1}+N_{2}}
\end{array}\right], \quad \mathbf{u}=\left[\begin{array}{c}
u_{0} \\
u_{1} \\
\\
u_{N_{1}-1} \\
u_{N_{1}} \\
u_{N_{1}+1} \\
\\
u_{N_{1}+N_{2}-1}
\end{array}\right],
$$

$$
\begin{aligned}
& \Xi_{i}=\left[\begin{array}{ccccc}
C_{i} B_{i} & & & & \\
C_{i} A_{i} B_{i} & C_{i} B_{i} & & & \\
C_{i} A_{i}^{2} B_{i} & C_{i} A_{i} B_{i} & C_{i} B_{i} & & \\
& \mathrm{M} & & \mathrm{O} & \\
C_{i} A_{i}^{N_{i}-1} B_{i} & C_{i} A_{i}^{N_{i}-2} B_{i} & C_{i} A_{i}^{N_{i}-3} B_{i} & \mathrm{~L} & C_{i} B_{i}
\end{array}\right] \\
& \Phi(j, k)=C_{2} A_{2}^{j} A_{1}^{N_{1}-k} B_{1}, j=1 \ldots N_{2}, k=1 \ldots N_{1} \\
& \Theta_{i}(j)=C_{i} A_{i}^{j}, \quad j=1 \ldots N_{i}
\end{aligned}
$$

$$
\Psi(j)=C_{2} A_{2}^{j} A_{1}^{N_{1}}, \quad j=1 \ldots N_{2} .
$$

The initial error vector $\tilde{x}_{01}$ contains the error of the state variables before the injection, while $\Delta x_{i d}$ is the difference between the ideal state variables in steady state before and after the reconfiguration of the system. The state error variables after $N_{1}+N_{2}$ steps can be expressed as follows:

$$
\tilde{x}_{N_{1}+N_{2}}=\left[A_{2}^{N_{2}} \Gamma_{1} \mid \Gamma_{2}\right] \mathbf{u}+\left[A_{2}^{N_{2}} A_{1}^{N_{1}} \mid A_{2}^{N_{2}}\right]\left[\begin{array}{c}
\tilde{x}_{01} \\
\Delta x_{i d}
\end{array}\right],
$$

where

$$
\Gamma_{i}=\left[A_{i}^{N_{i}-1} B_{i}\left|A_{i}^{N_{i}-2} B_{i}\right| \mathrm{L}\left|A_{i} B_{i}\right| B_{i}\right]
$$

The goal is to minimize the effect of the transient (i.e. the power of the output error), and the state error after the injection. The power of the combined vector $\left[\begin{array}{c}\mathbf{y} \\ \tilde{x}_{N_{1}+N_{2}}\end{array}\right]$ can easily be minimized by solving the following equation in leastsquares sense:

$0=\left[\begin{array}{c}\mathbf{y} \\ \tilde{x}_{N_{1}+N_{2}}\end{array}\right]=\left[\begin{array}{cc}{\left[\begin{array}{cc}\Xi_{1} & \mathbf{0} \\ \Phi & \Xi_{2}\end{array}\right]} \\ A_{2}^{N_{2}} \Gamma_{1} \mid \Gamma_{2}\end{array}\right] \mathbf{u}+\left[\begin{array}{c}{\left[\begin{array}{cc}\Theta_{1} & \mathbf{0} \\ \Psi & \Theta_{2}\end{array}\right]} \\ A_{2}^{N_{2}} A_{1}^{N_{1} \mid} A_{2}^{N_{2}}\end{array}\right]\left[\begin{array}{c}\tilde{x}_{01} \\ \Delta x_{i d}\end{array}\right]$

The least-square solution is

$$
\mathbf{u}=\left[\begin{array}{cc}
{\left[\begin{array}{cc}
\Xi_{1} & \mathbf{0} \\
\Phi & \Xi_{2}
\end{array}\right]} \\
A_{2}^{N_{2}} \Gamma_{1} \mid \Gamma_{2}
\end{array}\right]\left[\begin{array}{cc}
{\left[\begin{array}{cc}
\Theta_{1} & \mathbf{0} \\
\Psi & \Theta_{2}
\end{array}\right]} \\
A_{2}^{N_{2}} A_{1}^{N_{1}} \mid A_{2}^{N_{2}}
\end{array}\right]\left[\begin{array}{c}
\tilde{x}_{01} \\
\Delta x_{i d}
\end{array}\right] \equiv \mathbf{S}\left[\begin{array}{c}
\tilde{x}_{01} \\
\Delta x_{i d}
\end{array}\right]
$$

where $X^{\#}$ denotes the pseudo-invert of $X$.

Notes:

- If the reconfiguration is made deliberately (mode change), and both the old and new plant models are known a priory then the matrix $S$ in (14) can be computed off-line. In this case the on-line computational complexity is quite low and proportional to the number of states and the length of the injected signal.

- If the modes are not known a priory then (14) must be computed on-line. The computational complexity is much higher in this case; see the next section for an example. 
- The effect of the output error and state mismatch can be weighted to provide a not equally balanced solution.

- The least-squares formulation doesn't enable to set limits on the input signal thus the amplitude of the solution can be higher than a given physical limit. In this case other 'guard-states' are introduced (e.g. delayed or filtered version of the input), the weighted power of which is also taken into consideration in the solution. By the adjustment of this weight factor the amplitude of the solution can be arbitrarily limited. Note that this is not the optimal solution.

- Although no analytical sensitivity analysis has been made yet, simulation examples show that the transient rejection is still working if the used model is not perfect, but describes the behavior of the plant reasonably well.

- This approach takes into consideration the dynamics of the plant itself, and not that of the full closed loop. The idea behind this simplification is that the injected transient signal is usually shorter than the dominant time constant of the system. Of course, the same calculations could be made for the whole closed loop.

\section{SIMULATION RESULTS}

As an example the roll control of a simulated airplane has been simulated ([9], P7.5). The plant (airplane) changed at time instant 50s. The original aircraft has two identical poles at $s=-4$, while the reconfigured dynamics have poles at $s=-3$ and $s=-5$. The controller was not changed in the example. Fig. 3 shows the transient behavior without transient control, and with the introduction of two different anti-transient signals. The a posteriori control used $N_{1}=0$ and $N_{2}=100$, while the a priori signal was designed with values $N_{1}=40$ and $N_{2}=100$. It is clearly visible that the anti-transient signal decreased both the amplitude and the length of the undesired reconfiguration transient.

Table I contains additional information on different antitransient signals: the output error power and the computational times. $T_{\text {cacl1 }}$ and $T_{\text {calc2 }}$ are the computational times with and without the calculation of matrix $S$ in (14), respectively, measured on a Pentium III class computer in Matlab.

Table I

Properties of the Anti-Transient Control signals for the roll-control example with different parameters.

\begin{tabular}{c|c|c|c|c}
\hline $\boldsymbol{N}_{\mathbf{1}}$ & $\boldsymbol{N}_{\mathbf{2}}$ & Output error power & $\boldsymbol{T}_{\text {calc1 }}$ & $\boldsymbol{T}_{\text {calc2 }}$ \\
\hline 0 & 0 & 1.81 & 0 & 0 \\
\hline 0 & 20 & 0.93 & $0.1 \mathrm{~s}$ & $0.08 \mathrm{~ms}$ \\
\hline 0 & 40 & 0.48 & $0.2 \mathrm{~s}$ & $0.10 \mathrm{~ms}$ \\
\hline 0 & 100 & 0.20 & $0.9 \mathrm{~s}$ & $0.12 \mathrm{~ms}$ \\
\hline 10 & 20 & 0.64 & $0.1 \mathrm{~s}$ & $0.08 \mathrm{~ms}$ \\
\hline 10 & 40 & 0.32 & $0.2 \mathrm{~s}$ & $0.11 \mathrm{~ms}$ \\
\hline 20 & 100 & 0.08 & $1.1 \mathrm{~s}$ & $0.13 \mathrm{~ms}$ \\
\hline 40 & 100 & 0.04 & $1.2 \mathrm{~s}$ & $0.14 \mathrm{~ms}$ \\
\hline
\end{tabular}
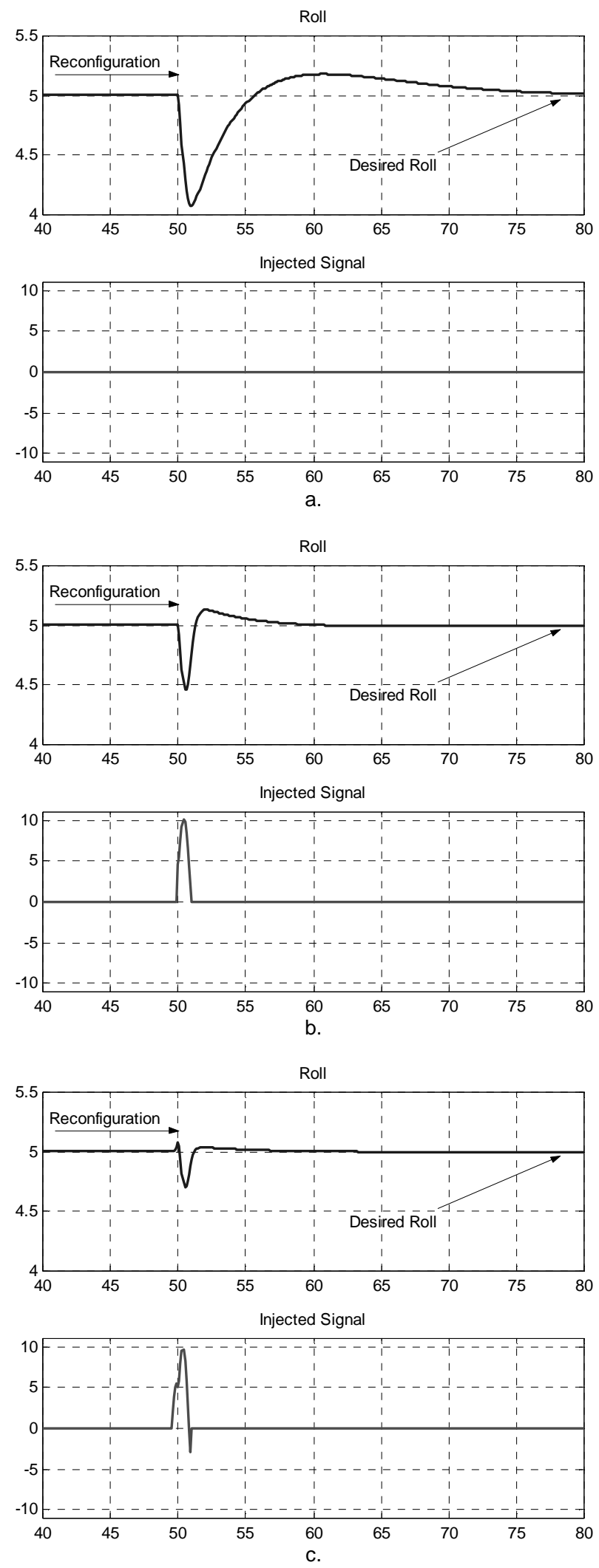

Fig.3. Simulated anti-transient control of an airplane. a. no injection, b. a posteriori injection, c. a priori injection 


\section{CONCLUSION}

A new transient management technique was proposed for closed loop control systems. The active transient control reduces transients in the control loop caused by the plant reconfiguration, by injecting additional anti-transient signal into the system. The problem was addressed as a least-squares optimization problem. Combined with appropriate controller reconfiguration methods simultaneous controller-plant reconfigurations can also be handled. Simulation examples illustrated the effectiveness of the proposed anti-transient method.

\section{ACKNOWLEDGMENT}

This research was sponsored by DARPA (US) (F33615-99-C3611), the Hungarian Ministry of Education (OM-FKFP 0654/2000) and by the Hungarian National Scientific Research Fund (OTKA) under contact number F 033055.

\section{REFERENCES}

[1] Grossman, R.L., A. Nerode, A.P. Ravn, and H. Rischel, Eds., Hybrid Systems, Lecture Notes in Computer Science, no. 736, New York: Springer Verlag, 1993.

[2] Sztipanovits, J., D.M. Wilkes, G. Karsai, Cs. Biegl, L.E. Lynd, "The Multigraph and structural adaptivity," IEEE Transactions on Signal Processing, Vol.41, No.8, pp. 2695-2716, Aug. 1993.

[3] Narendra, K. S., J. Balakrishman, "Adaptive Control Using Multiple Models," IEEE Trans. on Automatic Control, Vol. 42, No.2, pp.171187, Febr. 1997.

[4] Simon, Gy., T. Kovácsházy, G. Péceli, "Transients in Reconfigurable Control Loops," IEEE Instrumentation and Measurement Technology Conference, IMTC/2000, Baltimore, Maryland, USA, May 1-4, 2000 Vol. 3. pp. 1333-1337.

[5] Kovácsházy, T., G. Péceli, Gy. Simon, "Transients in Reconfigurable Signal Processing Channels," IEEE Instrumentation and Measurement Technology Conference, IMTC/2000, Baltimore, Maryland, USA, May 1-4, 2000, Vol. 1. pp. 241-246.

[6] Zhang,Y, J. Juang, "Design of Integrated Fault Detection, Diagnosis and Reconfigurable Control Systems," Proc. of the $38^{\text {th }}$ Conference on Decision \& Control, Phoenix, Arizona, USA, Dec. 1999, pp. 3587 3592 .

[7] Sun, J., "A Modified Model Reference Adaptive Control Scheme for Improved Transient Performance," IEEE Trans. on Automatic Control, Vol. 38, No. 8, pp. 1255-1259, Aug. 1993.

[8] Valimaki, V., T.I. Laakso, "Suppression of transients in variable recursive filters with a novel and efficient cancellation method," IEEE Trans. on Signal Processing, Vol. 46, (1998), pp. 3408-3414.

[9]. Shinners, S.M., Advanced Modern Control System Theory and Design. John Wiley \& Sons, Inc, New York, 1988. 